\title{
Dossier « Le réveil du dodo III » Les éthiques environnementales
}

\author{
Catherine Larrère \\ Philosophe, Université Paris 1 Panthéon-Sorbonne, UFR10, 17 rue de la Sorbonne, 75231 Paris cedex 05, France
}

\author{
Mots-clés : \\ éthique \\ environnementale ; \\ écologie ; \\ valeur intrinsèque ; \\ communauté biotique
}

\section{Keywords:}

environmental ethics; ecology;

intrinsic value;

biotic communities

\begin{abstract}
Résumé - L'article se propose d'étudier les grands courants de l'éthique environnementale (biocentrisme, écocentrisme, pragmatisme), en les replaçant dans leur contexte (les années 1970 et l'intérêt pour les questions environnementales), en en faisant apparaître les caractéristiques principales (valeur intrinsèque, communauté biotique, multiplicité des valeurs environnementales), en prenant en considération les difficultés qu'ils rencontrent (une approche trop individualisée pour le biocentrisme, les risques du holisme pour l'écocentrisme, l'oubli de la nature pour le pragmatisme). L'article en vient finalement à un examen des objections politiques qui ont été faites à ces courants (sur la question du pluralisme, notamment), avant de conclure en faveur d'une éthique relationnelle ouverte au débat public.
\end{abstract}

\begin{abstract}
Trends in environmental ethics. This article investigates the main trends in environmental ethics (biocentrism, ecocentrism, pragmatism). It starts by outlining the context in which these various ethics emerged, i.e. concern for environmental issues in the 70s. It then highlights the main characteristics of each trend (intrinsic value, biotic community, diversity of environmental values) and points out the main difficulties each trend faces (an overly individualistic approach in the case of biocentrism, risks of holism with ecocentrism, forgetting nature with pragmatism). Last, it examines the political objections which were raised about these ethics, especially regarding pluralism. The papers concludes in favour of a relational ethics capable of advertising the "good news" of ecology, i.e. that we live in a world in which everything is interconnected, and open to a pluralistic and democratic discussion rather than enforcing some scientific truth.
\end{abstract}

\section{Introduction}

«Is there a need for a new, an environmental, ethic?». En 1973, un philosophe australien, Richard Routley (qui allait ensuite se faire appeler Richard Sylvan), présentait, à un congrès international de philosophie à Sofia, en Bulgarie, une communication qui allait renouveler la réflexion morale en faisant entrer la nature dans le domaine de la moralité. L'idée était qu'il y a de bonnes et de mauvaises façons de se conduire dans la nature, que nos rapports avec celle-ci ont d'autres limites que celles de notre puissance technique, que nous avons à son égard des devoirs, qu'elle a peut-être des droits, que la nature, donc, a une valeur morale (Routley, 1973) ${ }^{1}$.

\footnotetext{
Auteur correspondant : c.larrere@wanadoo.fr

* Cf. dans ce même numéro la présentation, par la Rédaction, de ce dossier et de son contenu.

1 Cette communication a été présentée, le 17 mars 2009, en introduction au colloque «Le réveil du dodo III, $3^{\text {es }}$ journées francophones des sciences de la conservation de la biodiversité », qui avait pour thème : "Peut-on faire de la biologie de
}

L'idée, sans doute, n'était pas complètement neuve, et le souci qui l'inspirait n'était pas inédit. On peut faire remonter au XIXe siècle une attention portée à la nature qui est inséparable des transformations rapides de l'environnement consécutives à l'industrialisation. Ce sont les sociétés industrielles, et elles seules, qui ont formulé et donné sens à un projet de protection de la nature, destiné à mettre des espaces à l'abri du développement économique et industriel. Le $1^{\text {er }}$ mars 1872 est établi, aux États-Unis, le premier parc national, le Yellowstone, alors qu'en France, à partir de 1853, les "séries artistiques » de Fontainebleau font l'objet de mesures de protection. Si ce mouvement de protection de la nature a pris, aux États-Unis, une ampleur et une importance considérables, cela tient sans doute à la rapidité et à la violence de la transformation des terres et des espaces appropriés par les colons : ceux-ci ont fait, en moins de cent ans, ce qu'il avait fallu des siècles aux Européens pour accomplir. Ils ont été ainsi brutalement mis en face des

la conservation sans les sciences de l'homme et de la société? » (Université Montpellier 2, IFR119, Montpellier). 
résultats de leurs actions. Lorsque disparaît la frontière, les colons américains peuvent se dire qu'avec celle-ci, une partie de leur identité est mise en cause et qu'il leur faut préserver une nature contre laquelle, mais tout autant avec laquelle, l'identité américaine s'est affirmée. Il existe donc toute une tradition qui puise dans le romantisme américain (particulièrement chez Thoreau et Emerson) un amour d'une nature sauvage (wilderness) qu'il faut respecter et préserver. John Muir, fondateur du Sierra Club, qui reste l'une des plus puissantes organisations environnementales américaines, est l'un des représentants les plus connus d'un mouvement de protection d'espaces naturels qui aboutit à l'adoption, en 1964, du Wilderness Act qui régit, aux États-Unis, la protection de la nature (Nash, 1967).

Après la Deuxième Guerre mondiale, s'il est de plus en plus question de mettre des espaces naturels à l'abri des transformations industrielles, ce sont également les effets généraux de ces transformations qui sont remarqués et redoutés : extension et multiplication des pollutions, épuisement des ressources, disparitions ou destructions irréversibles. Alors que l'on s'emploie à reconstruire les économies européennes, et que l'on se préoccupe de l'accès du reste du monde à un niveau de richesse et de bien-être comparables à celui des pays occidentaux, on commence à s'inquiéter de la possibilité de poursuivre indéfiniment le même mode de développement économique : toute une réflexion s'élabore de la sorte, marquée notamment par le rapport Meadows (1972) sur les limites de la croissance. En 1962, une scientifique américaine, spécialiste de biologie marine, Rachel Carson, publie un livre, Silent Spring, qui, pour beaucoup, lance le mouvement environnemental aux États-Unis (Carson, 1962). Elle y montre les effets cumulatifs et dévastateurs de l'emploi des pesticides, tout particulièrement du DDT. L'idée s'impose qu'il ne s'agit pas de phénomènes épars, mais que toutes ces conséquences de nos interventions techniques dans la nature (auxquelles s'ajoute le poids grandissant d'une humanité en pleine croissance démographique) se réunissent et se globalisent en une crise environnementale. On peut donc chercher à remonter aux racines de cette crise et tenter d'en déceler l'origine commune dans un certain rapport à la nature. En 1967, un historien des techniques (et plus précisément des techniques médiévales), Lynn White, Jr., publie, dans la prestigieuse revue Science, un article qui devait avoir un immense retentissement : « The historical roots of our ecological crisis » (White, Jr., 1967). Il y rendait le christianisme - et la Bible, qui lui sert de référence - responsables de la crise environnementale : en créant l'Homme à son image, Dieu le met à part du reste de la Création - qui n'est plus, dès lors, qu'un instrument au service des besoins humains.

Le terrain était ainsi préparé pour une réflexion philosophique sur ces questions environnementales ou écolo- giques et plusieurs interventions ou publications allèrent dans ce sens. En 1973, paraissaient simultanément, outre l'article de Richard Routley, un article d'Arne Naess qui devait lancer l'expression de « deep ecology » et un de Peter Singer sur la libération animale, et, en 1974, un article de Georges Canguilhem intitulé «La question de l'écologie » (Naess, 1973; Singer, 1973; Canguilhem, 1974).

$\mathrm{Si}$, à la convergence de ces textes, se dessinent les contours philosophiques d'une réflexion émergente sur les questions d'environnement, seul l'article de Routley pose directement et centralement la question d'une éthique de la nature ou de l'environnement ${ }^{2}$. On peut ainsi considérer que cet article marque le début d'une réflexion philosophique et morale sur l'environnement et les rapports de l'homme à la nature qui, dans les pays de langue anglaise (Angleterre, Amérique du Nord, Australie), s'est développée en un courant d'éthique environnementale ayant ses différentes tendances, ses revues scientifiques à comité de lecture, ses associations et ses congrès ${ }^{3}$. Dans cet article pionnier, l'auteur construit un cas fictif, celui du dernier homme à survivre sur terre (après une catastrophe mondiale), Mr Last Man. Il s'emploie, avant de disparaître, à détruire tout ce qui l'entoure : plantes, animaux... Comment évaluer ce qu'il fait ? Si l'on s'en tient à l'éthique dominante dans le monde occidental, où il n'y a de droits et de devoirs qu'entre les hommes, il ne fait rien de mal, puisqu'il ne lèse personne. Mais si l'on considère qu'il y a des valeurs dans la nature, que nous avons des devoirs à leur égard, alors son acte est moralement condamnable ${ }^{4}$.

\section{Biocentrisme}

L'éthique environnementale, qui s'est développée dans la foulée de cet article, s'est élaborée autour de l'idée de la valeur intrinsèque, celle des entités naturelles, ou de la nature comme un tout. L'expression de « valeur intrinsèque » se trouve chez Kant : a une valeur intrinsèque tout ce qui doit être traité comme une « fin en soi », c'est-à-dire l'humanité et, plus généralement, tout être raisonnable. Tout le reste n'est considéré que comme un moyen, comme une valeur instrumentale : « Les êtres dont l'existence dépend, à vrai dire, non pas de notre volonté mais de la nature, n'ont cependant, quand ce sont des êtres dépourvus de raison, qu'une valeur relative, celle de moyens, et voilà pourquoi on les nomme

\footnotetext{
${ }^{2}$ L'article de Naess a une orientation plus politique, et les développements ultérieurs de sa réflexion (Naess, 1989) intègrent la dimension morale dans une réflexion philosophique d'ensemble.

3 Pour une présentation de ces réflexions morales, voir Larrère (1997), Light et Rolston III (2003), Afeissa (2007).

4 Un cas comparable est celui de Robinson Crusoé sur le point de quitter son île. Voir Midgley (1985, pp. 174-191).
} 
des choses ${ }^{5}$. » L'éthique environnementale va nommer « anthropocentrique » cette position qui ne reconnaît de dignité morale qu'aux humains et laisse, en dehors de son champ, tout le reste, c'est-à-dire la nature, vue comme un ensemble de ressources. L'ambition de l'éthique environnementale est au contraire de montrer que les entités naturelles ont une dignité morale, qu'elles sont des valeurs intrinsèques.

L'idée est que, là où il y a des moyens, il y a nécessairement des fins. Or, tous les organismes vivants, du plus simple au plus complexe, qu'il s'agisse d'animaux (même dépourvus de sensibilité), de végétaux ou d'organismes monocellulaires, tous déploient, pour se conserver dans l'existence et se reproduire, des stratégies adaptatives complexes, qui sont autant de moyens au service d'une fin. Il y a donc des fins dans la nature. On peut considérer tout être vivant comme l'équivalent fonctionnel d'un ensemble d'actes intentionnels, comme une « fin en soi » : « Les organismes - affirme Rolston III (1987, p. 269), un des théoriciens de la valeur intrinsèquevalorisent ces ressources de façon instrumentale, parce qu'ils s'accordent à eux-mêmes, à la forme de vie qu'ils sont, une valeur intrinsèque. » À l'opposition entre les personnes humaines et les choses, caractéristique de l'anthropocentrisme, se substitue une multiplicité d'individualités téléonomiques qui peuvent toutes prétendre, au même titre, être des fins en soi, et donc avoir une valeur intrinsèque (Taylor, 1981 et 1986; Rolston III, 1994a et b ; Callicott, 1999a). Tout individu vivant est, à égalité avec tout autre, digne de considération morale : c'est ce qu'on appelle le biocentrisme.

L'éthique environnementale biocentrique reconnaît ainsi un vouloir-vivre (une infinité de vouloir-vivre individuels) à l'œuvre dans la nature entière, et transfère à la vie, à tout ce qui est vivant, la dignité morale que l'éthique kantienne accorde aux êtres libres. Il s'agit donc d'une éthique du respect de la nature, dont Paul Taylor (1986) détaille les principes : (1) Tous les êtres vivants ont un statut égal ; (2) On ne peut traiter une valeur intrinsèque comme un simple moyen; (3) Chaque entité individuelle a droit à la protection ; (4) Il s'agit bien d'une affaire de principe, d'un principe moral. L'éthique du respect de la nature est donc une éthique déontologique, qui évalue les actions morales suivant qu'elles respectent ou non des principes moraux, nullement en anticipant des conséquences. C'est cet aspect déontologique qui peut expliquer le succès de l'éthique du respect de la nature. Elle implique une véritable conversion morale : il s'agit de se déprendre de l'égoïsme des conceptions morales traditionnelles, anthropocentriques (leurs détracteurs parlent à ce sujet de «chauvinisme humain »), pour découvrir la valeur de tout ce qui nous entoure. De quel droit ne

5 Kant, E., 1985. Fondements de la métaphysique des mœurs, IIe section, in CEuvres philosophiques, Paris, Gallimard, t. II, p. 294. reconnaissons-nous de valeur qu'à nous-mêmes, nous les humains?

La reconnaissance de la valeur intrinsèque passe par une sorte de sursaut moral, une attention au vivant qui a rapidement gagné des adeptes. La valeur intrinsèque est devenue le cri de ralliement de nombreux militants de la protection de la nature :

«C'est la supposition qu'ils défendent des valeurs réelles, existant objectivement dans la nature, qui motive beaucoup de militants environnementalistes, parmi lesquels des militants d'Earth first!, des membres de Greenpeace et de la Wilderness Society [...]. Il existe des éléments concordants pour penser que la croyance en l'existence de valeurs intrinsèques dans la nature joue un rôle de plus en plus important dans la formation des attitudes et des politiques environnementales dans le monde entier. » (Preston, 1998, pp. 410-411.)

La Convention sur la diversité biologique (Rio, 1992), en affirmant, dans son article 1, la «valeur intrinsèque de la biodiversité », porte témoignage de l'importance prise par la référence à la valeur intrinsèque. On en retrouve aussi la marque dans les différents textes législatifs qui règlent la protection des espèces : ils impliquent le plus souvent l'interdiction de tout prélèvement individuel des composantes de ces espèces. Cette attention à l'entité individuelle est caractéristique du biocentrisme.

Reconnaître une valeur intrinsèque à chaque entité vivante, c'est admettre qu'elle existe d'une façon telle que l'on ne peut en disposer de façon arbitraire, qu'elle ne peut être à volonté remplacée par un équivalent. Cela ne conduit pas à s'interdire toute intervention dans la nature qui risquerait de tuer des êtres vivants (ce serait impossible), mais à en rendre nécessaire la justification. Aussi longtemps que l'anthropocentrisme est dominant (c'està-dire que les êtres humains sont considérés comme les seules fins en soi, dignes d'être moralement considérées), la charge de la preuve, là où la diversité biologique est en danger, revient aux protecteurs de la nature : ils doivent prouver que telle ou telle perte de diversité biologique entraînera plus de coûts que d'avantages pour les populations humaines. Se ranger au biocentrisme conduirait à inverser la charge de la preuve : ce seraient ceux qui proposeraient de nouvelles activités, potentiellement dangereuses, qui devraient apporter la preuve que les bénéfices qu'on en retirerait seraient si importants qu'ils justifieraient que l'on sacrifie quelque chose qui a une valeur intrinsèque.

Mais, si les adeptes de l'éthique biocentrique justifient ainsi son utilité pratique, on peut cependant avoir quelque doute sur la réalité de celle-ci. Comment une éthique qui accorde une valeur égale à toutes les entités vivantes peut-elle répondre aux besoins d'une politique de protection de la nature qui passe par des choix entre plusieurs scénarios possibles, ce qui implique que l'on puisse hiérarchiser les valeurs? En outre, protéger 
la nature, ce n'est pas tant sauvegarder des individus que des populations, et prendre en considération des ensembles complexes (écosystèmes, ensembles d'écosystèmes, paysages) où se lient le vivant et le non-vivant (auquel l'éthique biocentrique ne reconnaît pas de valeur). On comprend donc le besoin d'une éthique qui ne se contente pas d'énoncer quelques affirmations de principe (qui se traduisent essentiellement par des interdictions), mais permette de diriger pratiquement les actions de protection.

\section{Écocentrisme}

Certains environnementalistes, comme Baird Callicott, considèrent ainsi qu'il faut accorder de la valeur non pas à des éléments séparés, mais à l'ensemble qu'ils forment, à la "communauté biotique ». Cette approche, dite "écocentrique ", se réclame d'un forestier américain de la première moitié $\mathrm{du} X \mathrm{X}^{\mathrm{e}}$ siècle, Aldo Leopold. Dans un livre écrit à la fin de sa vie, $A$ Sand County Almanac, Leopold, dans la tradition américaine des récits de nature (dont Thoreau, avec Walden, est l'initiateur), enchaîne, en suivant les mois de l'année, une série d'historiettes, ou de vignettes, où il raconte ses promenades matinales dans son domaine du Wisconsin (le « comté des sables »), les animaux qu'il y rencontre, tous usagers d'un même territoire. Ces récits vivants et attachants débouchent sur la présentation d'une éthique environnementale (qu'Aldo Leopold nomme Land Ethic). Une formule la résume : "Une chose est juste lorsqu'elle tend à préserver l'intégrité, la stabilité et la beauté de la communauté biotique. Elle est injuste lorsqu'elle tend à l'inverse » (Leopold, 1995, p. 283).

Un des récits les plus célèbres de l'Almanach, «Penser comme une montagne » (Leopold, 1995), permet d'en saisir le sens. Leopold s'y présente en chasseur; mais le cri de la louve sur laquelle il vient de tirer anéantit ses certitudes sur la nécessaire disparition des loups. Au détour de cette fable, Leopold se livre à une critique de la politique d'extermination des «nuisibles » décidée par l'Office américain de gestion de la faune sauvage, politique à laquelle il avait lui-même commencé par participer activement et qui avait conduit à la disparition des loups dans de nombreux États américains. Une extermination tout à l'avantage des chasseurs, avait-on pensé, mais la pullulation des cerfs et des daims qui s'était ensuivie avait été de courte durée, et avait en revanche entraîné des dommages écologiques (surpâturage, dégradation des pentes) de longue durée. «Penser comme une montagne » met en scène la situation et montre ainsi comment l'éleveur et son troupeau, le chasseur et ses proies, tous ont intérêt - du moins le croient-ils - à la disparition du loup. Mais, du point de vue de ce bien commun qu'est pour eux la montagne, avec ses ressources - arbres et herbe -, ils se trompent, ils ont la vue trop courte. Le loup a sa place dans la communauté biotique qui vit de la montagne. La prospérité des troupeaux et celle du gibier en dépendent à long terme. Leopold découvre ainsi le niveau qui intègre les points de vue, assignant à chacun sa place : c'est celui de la montagne, qui « sait » que, sans les loups, les cerfs proliféreront et mettront ses pentes à mal.

À la différence du biocentrisme, qui insiste sur la valeur propre, intrinsèque, de chaque entité vivante, considérée isolément, l'éthique de Leopold met l'accent sur l'interdépendance des éléments et leur commune appartenance à un ensemble, celui de la "communauté biotique ». Cette éthique, que l'on a pu dire «holiste » (par opposition à l'individualisme du biocentrisme), fait procéder les devoirs ou les obligations de l'appartenance à une totalité (que représente symboliquement la montagne) qui englobe ses membres. Ceux-ci n'ont pas de valeur en eux-mêmes, indépendamment de la place qu'ils occupent dans l'ensemble et qui leur assigne leur valeur. L'homme n'est donc pas extérieur à la nature, il en fait partie : il est membre, au même titre que les loups ou les cerfs, de la communauté biotique. Baird Callicott, qui s'est donné pour tâche de dégager les fondements philosophiques et les références scientifiques de la Land Ethic de Leopold, fait bien ressortir la double dimension, diachronique et synchronique, de cette solidarité des vivants $^{6}$. Diachroniquement, c'est la continuité de l'évolution telle qu'elle se dégage de l'enseignement de Darwin. «L'homme n'est qu'un compagnon-voyageur des autres espèces dans l'odyssée de l'évolution », affirme Leopold, qui insiste sur le retentissement moral, au niveau des sentiments, de cette proposition scientifique : «Cette découverte aurait dû nous donner, depuis le temps, un sentiment de fraternité avec les autres créatures; un désir de vivre et de laisser vivre ; un émerveillement devant la grandeur et la durée de l'entreprise biotique » (Leopold, 1995, p. 145). Synchroniquement, c'est l'enseignement de l'écologie, dont on pourrait dire que cela constitue la bonne nouvelle : nous faisons partie d'un tout dont les éléments sont interdépendants. Ce que «sait » la montagne, c'est ce qu'apprend l'écologie, dont les développements scientifiques furent contemporains de Leopold : la connaissance des chaînes trophiques, des échanges complexes d'énergie dans lesquels s'organise la poursuite de la $\mathrm{vie}^{7}$.

Comme l'explique Baird Callicott (1989b, p. 66), une éthique, c'est «la description de la structure de la communauté faite, de l'intérieur, par ses propres membres ». En ce qui concerne la communauté biotique,

\footnotetext{
6 " The land ethic rests upon three scientific cornerstones : (1) evolutionary and (2) ecological biology set in a background of (3) Copernican astronomy. » (Callicott, 1989a, p. 82.)

7 Voir la «pyramide de la terre " dans laquelle Leopold (1995, p. 271-278) expose, de façon imagée, ce réseau complexe d'échange et de circulation de l'énergie.
} 
cette description est donnée par l'écologie, ou par la théorie de l'évolution. Étroitement liée à un contenu scientifique, la Land Ethic s'expose donc à une constante révision. La formule de Leopold qui insiste sur l'intégrité et surtout sur la stabilité de la communauté biotique est datée : elle renvoie à un état de l'écologie qui met l'accent sur les équilibres de la nature, qu'il s'agisse de la notion de climax, telle que Clements a pu la présenter, comme l'état stable auquel parviennent les successions, ou de la vision thermodynamique de l'équilibre écosystémique exposée par Tansley. Or, les développements plus récents de l'écologie (écologie des perturbations, écologie des paysages) ont mis en cause cette prépondérance de l'équilibre, qui n'apparaît plus que comme un moment rare et précaire de dynamiques naturelles dont le régime le plus fréquent est celui des perturbations ${ }^{8}$. Callicott $(1999 b$, p. 138) s'est donc employé à actualiser la formule de Leopold, en tenant compte de ces transformations scientifiques, ce qui l'a amené à en présenter une nouvelle version : "Une chose est juste lorsqu'elle ne tend à perturber la communauté biotique qu'à des échelles temporelles et spatiales normales. Elle est injuste lorsqu'elle tend à l'inverse. »

La Land Ethic peut apparaître comme une redondance de l'écologie : il s'agit, selon Leopold (1995, p. 256), "de deux définitions différentes d'une même chose ". Ce que l'éthique apporte à l'écologie, c'est une modalité vécue : elle fait appel à des sentiments. Poursuivant son exploration des fondements conceptuels de la Land Ethic, Callicott fait ressortir tout ce qu'elle doit à la théorie des sentiments moraux, celle de Hume et de Smith, dont Darwin, le Darwin de La Filiation de l'homme, peut être considéré comme un continuateur. L'appartenance est vécue, ressentie comme un sentiment de fraternité avec les autres créatures, et toute la progression de l'Almanach d'un comté des sables, qui commence par des historiettes présentant des animaux avec juste ce qu'il faut d'anthropomorphisme pour nous les rendre attachants, est là pour éveiller ou réveiller nos sentiments de proximité avec la nature, dont nous découvrons ensuite le contenu descriptif qui les oriente. Comme le dit Leopold lui-même (ibid.), la Land Ethic est « en réalité un processus d'évolution écologique ». Ce sentiment de proximité, d'appartenance, que nous avons avec les autres membres de la communauté biotique, est une composante des comportements sociaux dont Darwin, dans La Filiation de l'homme, montre l'émergence. La Land Ethic peut donc être considérée comme une variante d'éthique évolutionniste : c'est, indique Leopold (1995, p. 257), « une sorte d'instinct communautaire en gestation $»$.

Comme les autres éthiques évolutionnistes, la Land Ethic se préoccupe de l'émergence des comportements sociaux («une éthique, explique Leopold [1995, p. 256],

\footnotetext{
${ }^{8}$ Sur la longue persistance, dans l'écologie scientifique, d'une conception des équilibres de la nature et sa récente remise en question, voir Blandin (2009).
}

distingue entre des formes sociales et asociales de conduite »). Mais la plupart des éthiques évolutionnistes (de Darwin à la sociobiologie) ne s'intéressent qu'aux comportements sociaux à l'intérieur d'une même espèce. La Land Ethic est plurispécifique. En élargissant «les frontières de la communauté de manière à y inclure le sol, l'eau, les plantes et les animaux, ou collectivement, la terre », la Land Ethic de Leopold ne dépasse pas seulement les frontières de l'humanité (celles, ordinaires, de la moralité), elle devient celle d'une communauté mixte, qui inclut diverses populations d'espèces différentes. Cela devrait enrichir l'appréhension de la variété des devoirs au sein de la communauté biotique. Comme le note Callicott, cette expression réunit le biologique et le social, en appliquant aux relations entre les vivants une métaphore tirée des rapports sociaux humains. Mais, de la façon dont Callicott applique la métaphore, cela conduit à appliquer un modèle essentiellement économique aux relations entre diverses espèces vivantes : «La communauté biotique, affirme Callicott (1999b, p. 72), est un système économique par excellence. » C'est insister sur l'interdépendance, mais c'est aussi se donner un modèle assez pauvre des relations possibles : s'agissant des communautés humaines, nous sommes généralement dans le dualisme, nous opposons égoïsme et altruisme, rivalité et coopération. La palette des relations au sein de la communauté mixte est beaucoup plus variée : prédation, rivalité, parasitisme, mutualisme, symbiose, coopération...

L'éthique biocentrique est déontologique; elle formule des normes universelles, principalement sous forme d'interdits : l'éthique du respect est essentiellement une éthique de la non-intervention. La Land Ethic de Leopold est conséquentialiste : la qualité d'une action ( « une chose est juste»-right...) se mesure à ses effets sur la communauté biotique («stabilité, intégrité, beauté »). Aussi Leopold (1995, p. 97) définit-il l'écologiste, ou le protecteur de la nature non pas comme celui qui s'abstient d'intervenir, mais comme celui-ci qui intervient à bon escient et ne craint pas de laisser une marque, ou une empreinte :

«J'ai lu de nombreuses définitions de ce qu'est un écologiste, et j'en ai moi-même écrit quelques-unes, mais je soupçonne que la meilleure d'entre elles ne s'écrit pas au stylo, mais à la cognée. La question est : à quoi pense un homme au moment où il coupe un arbre, ou au moment où il décide de ce qu'il doit couper? Un écologiste est quelqu'un qui a conscience, humblement, qu'à chaque coup de cognée, il inscrit sa signature sur la face de la terre. »

Mais ce qui fait l'avantage de la Land Ethic (elle permet de formuler des injonctions précises, et positives) l'ouvre également à la critique, car, du fait qu'elle vise un résultat global (au niveau de la communauté), elle ne prend pas nécessairement en compte la valeur des individus, dont les éthiques déontologiques font une question de principe. Comme toutes les éthiques conséquentialistes 
(notoirement l'utilitarisme), la Land Ethic s'expose à se voir reprocher de sacrifier les individus au bien commun ; mais, parce qu'elle est plurispécifique, elle s'expose en plus à se voir reprocher de ne pas accorder plus d'importance à une espèce qu'à une autre. Dans la communauté biotique, les humains se trouvent ainsi doublement exposés : comme individus, et comme espèce, que rien, a priori, ne justifie qu'on privilégie. Et ce, d'autant moins qu'ils sont les premiers à mettre en péril «l'intégrité, la stabilité et la beauté de la communauté biotique ${ }^{9}$ ».

\section{Pragmatisme}

Les difficultés que rencontrent les tentatives de franchir les limites habituelles de la moralité pour y inclure tous les vivants ou la communauté biotique expliquent que l'on ait pu tenter d'élaborer une éthique environnementale en revenant sur la condamnation de l'anthropocentrisme, qui avait été à l'origine des éthiques biocentriques ou écocentriques. Au biocentrisme, on peut en effet objecter son point de départ : une vision trop étroite de l'instrumentalisation, qui le conduit à rejeter l'anthropocentrisme avant d'en avoir examiné les ressources. Il n'est pas nécessaire d'opposer la valeur intrinsèque à la valeur instrumentale, il suffit de faire apparaître la diversité des valeurs instrumentales. L'utilité n'est pas seulement immédiate, ou matérielle; il faut prendre en considération qu'il y a un avenir, et des générations futures, qu'il y a des intérêts désintéressés, comme le sont les intérêts esthétiques ou cognitifs. Envisager la nature comme un ensemble de ressources, ce n'est pas nécessairement s'employer à la détruire : la nature nous fournit sans doute des biens (matières premières, produits agricoles...) que nous consommons en les détruisant, mais elle nous fournit aussi des services (pollinisation, recyclage, fixation des nitrates, régulation homéostatique), sans lesquels nous n'aurions pas accès à ces biens et qu'il est dans notre intérêt de maintenir en activité, nullement de faire disparaître. La même chose peut être dite de l'intérêt cognitif ou esthétique pour la nature. Si des scientifiques, comme les systématiciens, n'ont guère besoin d'éthique environnementale très élaborée, c'est qu'en défendant la nature, ils défendent leur objet de travail : Stephen Jay Gould (1996) raconte bien comment la disparition d'une espèce est une tragédie pour le naturaliste. De la même façon, ceux qui admirent la beauté de la nature, ou trouvent dans le sublime une expérience spirituelle qui élève leur âme, valorisent sans doute une expérience subjective qui leur est propre ; mais, ce faisant, ils ont besoin d'une nature intouchée sans laquelle cette expérience ne pourrait avoir lieu. Des programmes de protection de la nature sont parfaitement justifiables d'un point de vue anthropocentrique, et l'on peut, comme le fait Bryan Norton,

\footnotetext{
9 Callicott $(1999$ c) a répondu à ces critiques dans « Holistic environmental ethics and the problem of ecofascism».
}

estimer que c'est là le mode de justification le plus répandu chez les environnementalistes ${ }^{10}$. On peut ainsi distinguer, de l'anthropocentrisme réducteur dénoncé par les éthiques bio- ou écocentriques, un anthropocentrisme élargi ${ }^{11}$ tel que valoriser l'homme n'implique pas nécessairement de dévaloriser la nature.

En s'appuyant sur l'argument de bon sens selon lequel instrumentaliser la nature ne conduit pas nécessairement à la détruire, toute une réflexion d'inspiration pragmatiste s'est développée qui remet en cause la volonté de fonder l'éthique environnementale sur la valeur intrinsèque. Il est reproché à celle-ci de faire appel à une métaphysique lourde et de conduire à des positions sectaires. La quête de la valeur intrinsèque est la recherche d'une théorie unique, moniste de la valeur. Celle-ci risque d'autant moins d'être acceptée par le plus grand nombre qu'elle implique une interrogation métaphysique, une recherche du fondement, alors que la philosophie d'aujourd'hui a plutôt proclamé la fin de la métaphysique. À cette vision moniste et solitaire de la valeur, les pragmatistes opposent une vision pluraliste et relationnelle. Pourquoi, pour affirmer la valeur d'une forêt, faudrait-il s'en tenir à sa «valeur intrinsèque »? Il y a quantités de raisons de trouver une valeur à une forêt, quantité de façons de la valoriser. D'autre part, les valeurs ne sont pas isolées, il existe, pour chacun d'entre nous, des systèmes de valeurs qui sont liées les unes aux autres. Et cela, d'autant plus que les valeurs ne sont pas complètement indépendantes, elles n'existent que dans un contexte donné : la valeur (écologique) d'une plante n'est pas la même suivant qu'elle abonde dans un milieu ou que, en un autre endroit, elle n'est qu'un des rares spécimens de l'espèce à subsister encore (Weston, 1996).

À ce pluralisme des valeurs environnementales, il est un intérêt pratique. La recherche de la valeur intrinsèque est l'occasion de querelles métaphysiques qui divisent les environnementalistes, alors que la priorité devrait être à l'établissement d'un consensus sur les objectifs à poursuivre. Or, le pluralisme des valeurs ne s'oppose pas à ce consensus, au contraire, il le renforce. Des valorisations différentes peuvent très bien converger et, loin de conduire à des oppositions, renforcer les objectifs. À explorer les multiples raisons qui nous font attacher de la valeur à un lieu, on découvre d'autant plus d'arguments pour le protéger. Bien loin de rejeter les arguments en faveur de la protection de la nature que leur provenance anthropocentrique rendrait suspects (comme les environnementalistes figés dans leur rejet de l'anthropocentrisme sont accusés de le faire), Andrew Light (2003) comme Norton et les autres pragmatistes - en appelle à

\footnotetext{
10 «A majority of people, even a majority of environmentalists, seem to favor instrumental justifications for preserving species » (Norton, 1987, p. 175).

11 Parfois appelé «anthropocentrisme faible» («weak anthropocentrism ») : Hargrove (1989).
} 
toutes les justifications possibles du moment qu'elles ne sont pas compromises par des engagements intolérables (fascistes, par exemple) et qu'elles visent la même fin. Plus les justifications seront nombreuses, mieux cela sera! Aussi ne faut-il pas chercher à convertir à une théorie préexistante ceux qui se montreraient réticents devant cet objectif : il faut trouver des arguments qui soient recevables dans leurs propres conceptions morales et enrichir de la sorte l'argumentation en faveur de l'environnement.

Là où les tenants de la valeur intrinsèque ont tendance à rechercher l'argument massue qui emporte la conviction et ne réussissent qu'à être sectaires, les pragmatistes font ressortir les valeurs démocratiques du pluralisme : il autorise le compromis (car tous ceux qui s'entendent sur un même objectif sont prêts à abandonner une partie de leurs divergences) et il favorise la délibération, car, en rapprochant les arguments, on peut être amené à changer de raisons et à se rallier sur une base commune.

Ainsi, en réinstallant l'homme comme centre des valeurs, les pragmatistes n'abandonnent pas le souci de la nature. Mais ne se détournent-ils pas de ce qui peut être considéré comme l'enseignement principal des éthiques non anthropocentriques : nous ne sommes pas seuls au monde, les non-humains comptent aussi, pour euxmêmes?

\section{Conclusion}

Quand elles n'ont pas été complètement ignorées par les courants dominants de la réflexion morale, les éthiques environnementales ont rencontré de vives critiques. En étendant à toute la nature la sphère de la considération morale, leur a-t-on reproché, elles affaiblissent les droits humains préexistants tout en leur suscitant des concurrents aussi nombreux que redoutables (Thomas, 1998). On a même pu les accuser d'être fascistes, parce qu'elles autorisaient le sacrifice des individus à la communauté, et ce reproche était d'autant plus virulent que les critiques tendaient à fusionner, sous le nom collectif - et honni - de deep ecology, l'écocentrisme et le biocentrisme (Ferry, 1992).

Ces critiques, certainement exagérées, font cependant apparaître le problème : quelle place accorder aux éthiques environnementales au sein des théories morales déjà existantes? C'est la question du pluralisme : comment concilier des conceptions morales divergentes ou opposées, sans en imposer une au détriment des autres? Philippe Van Parijs propose qu'on assimile les éthiques environnementales à des positions religieuses, qui ne peuvent avoir de validité que dans la sphère privée. Ainsi écrit-il (Roose et Van Parijs, 1991, pp. 151-152) :

«Exiger un respect pour la Nature comme telle, indépendamment de l'intérêt que celle-ci présente pour l'homme [...] n'a pas d'autre poids, dans une société pluraliste, que le respect qu'une communauté de croyants exige pour les commandements de son Dieu : décisif s'il s'agit de déterminer le sens de sa vie ou la source de ses engagements, nul dès que l'on quitte le domaine de l'éthique privée pour établir par le débat public les règles qu'il est équitable que la société impose à tous ses membres. »

Et l'on pourrait peut-être envisager de la sorte l'éthique biocentrique. Elle réunit ceux qui reconnaissent la valeur intrinsèque des entités naturelles et demandent qu'on respecte la nature en des lieux (wilderness) où elle est laissée à son indépendance, à son autoréalisation. Faut-il considérer les éthiques environnementales comme le témoignage d'une nouvelle religion de la nature, à laquelle une société tolérante et pluraliste accorderait ses lieux de culte (les espaces naturels protégés) ? Ce serait, nous semble-t-il, gravement sous-estimer l'importance des questions environnementales, dans la vie de chacun de nous comme au niveau politique. Il ne s'agit pas seulement de laisser à quelques-uns la possibilité de vivre selon leurs convictions, il s'agit de prendre collectivement en charge nos rapports avec notre environnement. De cette entreprise collective témoignent aussi bien les conférences internationales et les sommets globaux que l'existence, au niveau des États mais aussi à des niveaux transnationaux (Europe) ou régionaux, de politiques et de programmes prenant en charge des questions environnementales. Il s'agit donc bien de déterminer, à la suite d'un débat public, les règles communes, pour tout ce qui concerne les politiques environnementales (et notamment celles qui organisent la protection de la nature). C'est bien de l'importance de ce débat public que témoignent notamment les éthiques pragmatistes, qui cherchent à explorer la pluralité des valeurs qui nous lient à notre environnement.

Dans ce débat public, les éthiques environnementales, dans leur diversité, font valoir trois intuitions : la première concerne la place des valeurs; la deuxième prend en compte le souci de la nature; la troisième touche à l'articulation de l'éthique et de la science.

La crise environnementale est en très grande partie le résultat des conséquences dommageables de nos actions techniques, elle met également en cause la poursuite, sans réajustements, de la croissance économique. Les éthiques environnementales mettent cependant en avant la conviction qu'il ne suffit pas d'apporter des remèdes techniques à des problèmes techniques et que la gestion de la crise ne peut être seulement économique : la réflexion sur la façon dont nous pouvons faire face à la crise engage des valeurs et a donc une dimension éthique ${ }^{12}$.

Cette dimension éthique englobe la nature et ses différentes composantes. L'idée est que la crise environnementale n'atteint pas seulement les hommes, mais qu'elle endommage ou fait disparaître d'autres êtres, à qui un

\footnotetext{
12 Cet aspect a été particulièrement développé par Dale Jamieson (2008).
} 
mal est fait, dont il faut tenir compte dans une estimation. Les hommes ne sont pas les seules valeurs que touche la crise. C'est à cette intuition que la théorie de la valeur intrinsèque essaie de donner une formulation rationnelle. Mais il n'est pas nécessaire de rejeter 1'anthropocentrisme pour lui donner raison. Nous pouvons valoriser autre chose que nous-mêmes. On a pu faire valoir que « l'échelle humaine des valeurs n'était pas seulement l'échelle des valeurs humaines » (Wiggins, 2000). Toutes les éthiques environnementales que nous avons présentées témoignent donc de la volonté d'inclure la nature dans notre souci moral : nous (les humains) ne sommes pas seuls au monde.

La troisième intuition touche à la façon dont peuvent s'accorder les exigences éthiques et les connaissances scientifiques. Contrairement à ce que l'on croit souvent, l'éthique appliquée ne consiste pas à mettre en pratique, dans un domaine particulier, des théories morales préexistantes. S'il en était ainsi, l'éthique environnementale ne serait pas une éthique appliquée, puisque, bien loin de faire appel à des théories morales déjà développées, elle fait ressentir le besoin de développer des théories morales nouvelles (et c'est bien pourquoi il y a des problèmes d'intégration de ces théories à un paysage moral préexistant). Ce qui caractérise une éthique appliquée, c'est qu'il s'agit de répondre à des problèmes, certes moraux, mais dont l'élaboration fait également appel à des connaissances scientifiques. C'est le cas en bioéthique ou en éthique médicale : on ne peut envisager les problèmes moraux posés par le clonage ou par la procréation médicale assistée qu'en s'appuyant sur une analyse scientifique de la question. C'est le cas aussi en éthique environnementale : les questions de protection de la nature ne peuvent être traitées indépendamment des connaissances apportées par la biologie de la conservation. Cela ne signifie pas qu'il y ait, de l'un à l'autre, de la connaissance scientifique aux positions morales, un rapport d'implication ou de dérivation logique, mais qu'il faut rechercher les positions qui concilient le mieux les deux domaines, ce qu'au XVIII ${ }^{\mathrm{e}}$ siècle on appelait un "rapport de convenance ». Certaines positions scientifiques et certaines positions éthiques s'accordent mieux ensemble que d'autres.

On peut trouver là le critère qui pourrait départager les différentes éthiques environnementales. La volonté d'inclure la nature dans notre souci moral a conduit à deux positions distinctes : le respect de l'altérité ; l'importance du relationnel. On peut reconnaître dans la nature un Autre, qui mérite le respect moral, dans sa distinction avec l'humain. Telle est la caractéristique des éthiques de la valeur intrinsèque, qui se retrouvent sur ce point en accord avec Hans Jonas (1990). On peut également insister sur tout ce qui nous lie à notre environnement, sur la multiplicité des relations que nous entretenons avec tous les êtres dont nous partageons la vie et qui la partagent avec nous et entre eux.
La première position s'accorde bien avec une vision dualiste de la nature, celle qui définit la nature par opposition à l'humain et entend l'altérité comme une extériorité. Les éthiques de la wilderness (ou du sauvage ${ }^{13}$ ) insistent sur cette dualité et cherchent à protéger la nature contre l'homme. C'est parce que nous sommes convaincus que cette conception d'une nature extérieure à l'homme est très largement remise en cause et qu'elle fait plutôt partie du problème que de la solution (car elle conduit à dramatiser l'opposition entre l'homme et la nature et provoque des accusations de misanthropie) que nous nous tournons vers les éthiques relationnelles, celles qui, insistant sur tout ce qui lie les hommes à l'environnement dont ils font partie, cherchent à renforcer positivement ces relations. Il n'est pas nécessaire de concevoir la nature sans l'homme. Au contraire.

Les éthiques pragmatistes sont des éthiques contextuelles et, pour cette raison, relationnelles : elles insistent sur l'insertion locale des problèmes environnementaux. On peut dire que, de ce point de vue, il y a une nette convergence entre le point de vue pragmatiste et l'approche écocentrique, telle qu'elle a pu être élaborée par Callicott. Norton comme Callicott se réfèrent à Leopold. La Land Ethic n'est pas une éthique dualiste, elle ne met pas l'homme à part de la nature, mais elle cherche les collaborations positives entre l'homme et la nature. Elle nous paraît, pour cette raison, très riche d'enseignements. Nous adopterons donc volontiers une position écocentrique : il s'agit de trouver les propositions morales qui correspondent à la «bonne nouvelle » de l'écologie (nous faisons partie d'un monde dont les éléments sont interdépendants). Ce faisant, nous ne nous reconnaissons pas dans les implications axiologiques exposées par Callicott : nous ne nous reconnaissons ni dans son holisme (l'unité de la communauté biotique n'est pas pour nous une donnée naturelle) ni dans l'idée qu'il y aurait une solution inscrite dans la nature aux différents conflits possibles entre les membres des communautés. La communauté est à constituer, et les solutions hiérarchisant les valeurs relèvent du débat public (non de l'application d'une formule préétablie ou de la seule étude scientifique). Si l'écocentrisme se révèle intéressant, c'est donc moins comme une position axiologique (accordant une valeur à la communauté comme telle) que comme une façon de nous situer dans le monde, dans un contexte dont nous faisons partie, mais au centre duquel nous ne sommes pas nécessairement situés, et où nous découvrons la multiplicité des réseaux d'interdépendance qui lient humains et non-humains ${ }^{14}$.

\footnotetext{
13 Représentées en France notamment par Jean-Claude Génot (2003).

14 Nous retrouvons des éléments de cette éthique contextuelle et relationnelle aussi bien dans les positions pragmatiques de Bruno Latour que dans les conceptions patrimoniales du développement durable.
} 


\section{Références}

Afeissa, H.S., 2007. Éthique de l'environnement: nature, valeur, respect, Paris, J. Vrin.

Blandin, P., 2009. De la protection de la nature au pilotage de la biodiversité, Paris, Quæ.

Callicott, J.B., 1989a. The conceptual Foundations of the Land Ethic, In Defense of the Land Ethic: Essays in Environmental Philosophy, Albany (NY), State University of New York Press, 75-100.

Callicott, J.B., 1989b. Elements of an environmental ethic, In Defense of the Land Ethic, op. cit., 63-74.

Callicott, J.B., 1999a. Intrinsic value in nature: A metaethical analysis, Beyond the Land Ethic: More Essays in Environmental Philosophy, Albany (NY), State University of New York Press, 239-261.

Callicott, J.B., 1999b. Do deconstructive ecology and sociobiology undermine the Leopold land ethic?, Beyond the Land Ethic, op. cit., 117-139.

Callicott, J.B., 1999c. Holistic environmental ethics and the problem of ecofascism, Beyond the Land Ethic, op. cit., 59-77.

Canguilhem, G., 1974. La question de l'écologie. La technique ou la vie, Dialogue, mars, 37-44. Article joint en annexe de Dagognet, F., 2000. Considérations sur l'idée de nature, Paris, J. Vrin, 183-189.

Carson, R.L., 1962. Silent Spring, Boston, Houghton Mifflin. Trad. fr. : Printemps silencieux, Marseille, Wildproject, 2009.

Ferry, L., 1992. Le Nouvel Ordre écologique, Paris, Grasset.

Génot, J.-C., 2003. Quelle éthique pour la nature?, Aix-enProvence, Édisud.

Gould, S.J., 1996 [éd. orig. 1993]. Un soir bien peu enchanté, Comme les huit doigts de la main : réflexions sur l'histoire naturelle, Paris, Le Seuil.

Hargrove, E.C., 1989. Foundations of Environmental Ethics, Englewood Cliffs (NJ), Prentice Hall.

Jamieson, D., 2008. Ethics and the Environment: An Introduction, Cambridge (UK) and New York, Cambridge University Press.

Jonas, Hans, 1990 [éd. orig. 1979]. Le Principe responsabilité : une éthique pour la civilisation technologique, Paris, Éditions du Cerf.

Kant, E., 1985. CEuvres philosophiques, t. II, Paris, Gallimard.

Larrère, C., 1997. Les Philosophies de l'environnement, Paris, PUF.

Leopold, A., 1995 [éd. orig. 1949]. Almanach d'un comté des sables, Paris, Aubier.

Light, A., 2003. The case for practical pluralism, in Light, A., Rolston III, H. (Eds), Environmental Ethics: An Anthology, Malden (MA), Blackwell Publishing, 229-247.

Light, A., Rolston III, H. (Eds), 2003. Environmental Ethics: An Anthology, op. cit.

Reçu le 4 novembre 2009. Accepté le 2 septembre 2010.
Meadows, D., 1972. The Limits to Growth: A Global Challenge. A Report for the Club of Rome Project on the Predicament of Mankind, Universe Books, New York.

Midgley, M., 1985. Evolution as a Religion, London and New York, Routledge.

Naess, A., 1973. The shallow and the deep, Long Range Ecology Movement: A summary, Inquiry, 16, 1-4, 95-100.

Naess, A., 2008 [ed. orig. angl. 1989]. Écologie, communauté et style de vie, [Paris], Éditions MF.

Nash, R.F., 1967. Wilderness and the American Mind, New Haven, Yale University Press.

Norton, B.G., 1987. Why Preserve Natural Variety?, Princeton (NJ), Princeton University Press.

Preston, C.J., 1998. Epistemology and intrinsic values: Norton and Callicott's critiques of Rolston, Environmental Ethics, 20, $4,405-422$.

Rolston III, H., 1987. Duties to ecosystems, in Callicott, B.J. (Ed.), Companion to a Sand County Almanac, Madison, The University of Wisconsin Press.

Rolston III, H., 1994a. Value in nature and the nature of value, reproduit in Light, A., Rolston III, H. (Eds), 2003. Environmental Ethics: An Anthology, Malden(MA), Blackwell Publishing, 143-153.

Rolston III, H., 1994b. Conserving Natural Value, New York, Columbia University Press.

Roose, F. de, Van Parijs, P., 1991. La Pensée écologique : essai d'inventaire à l'usage de ceux qui la pratiquent comme de ceux qui la craignent, Bruxelles, De Boeck Université.

Routley, R., 1973. Is there a need for a new, an environmental, ethic?, Proceedings of the XV World Congress of Philosophy, 17th to 22nd September 1973, Varna, Bulgaria, Sofia Press, 205-210.

Singer, P., 1973. Animal liberation, The New York Review of Books, 20,5 .

Singer, P., 1975. Animal Liberation: A New Ethics for our Treatment of Animals, New York, New York Review.

Taylor, P.W., 1981. The ethics of respect for nature, Environmental Ethics, 3, 3, 197-218.

Taylor, P.W., 1986. Respect for Nature: A Theory of Environmental Ethics, Princeton (NJ), Princeton University Press.

Thomas, Y., 1998. Le sujet de droit, la personne et la nature, Le Débat, 100, mai-août, 85-107.

Weston, A., 1996. Beyond intrinsic value: Pragmatism in environmental ethics, in Light, A., Katz, E. (Eds), Environmental Pragmatism, London and New York, Routledge, 285-306.

White, Jr., L., 1967. The historical roots of our ecological crisis, Science, 155, 3767, 1203-1207.

Wiggins, D., 2000. Nature, respect for nature, and the human scale of values, Proceedings of the Aristotelian Society, 100, 1, $1-32$. 